\title{
Correction: The novel microRNAs hsa-miR- nov7 and hsa-miR-nov3 are over-expressed in locally advanced breast cancer
}

\section{Deepak Poduval, Zuzana Sichmanova, Anne Hege Straume, Per Eystein Lønning, Stian Knappskog}

Fig 1 is missing part B. The authors have provided a corrected version here.

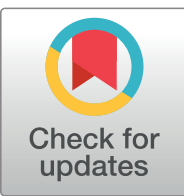

\section{G OPENACCESS}

Citation: Poduval D, Sichmanova Z, Straume AH, Lønning PE, Knappskog S (2021) Correction: The novel microRNAs hsa-miR-nov7 and hsa-miRnov3 are over-expressed in locally advanced breast cancer. PLoS ONE 16(6): e0253361. https://doi. org/10.1371/journal.pone.0253361

Published: June 10, 2021

Copyright: ๑ 2021 Poduval et al. This is an open access article distributed under the terms of the Creative Commons Attribution License, which permits unrestricted use, distribution, and reproduction in any medium, provided the original author and source are credited. 


\section{PLOS ONE}

A. hsa-miR-nov3

(i)

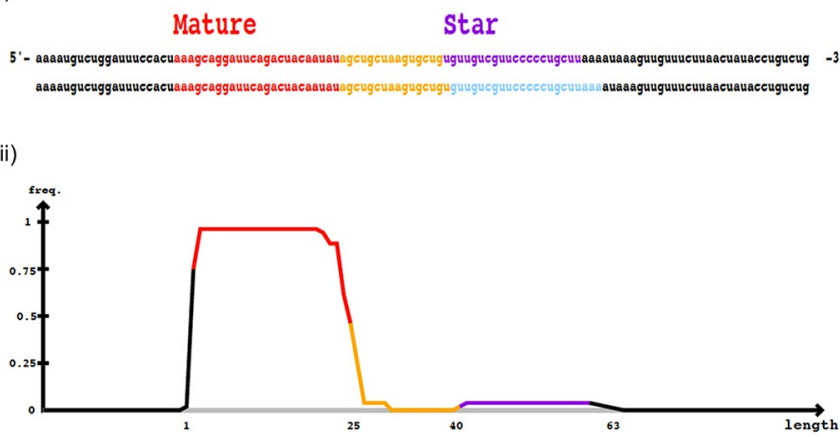

(iii)

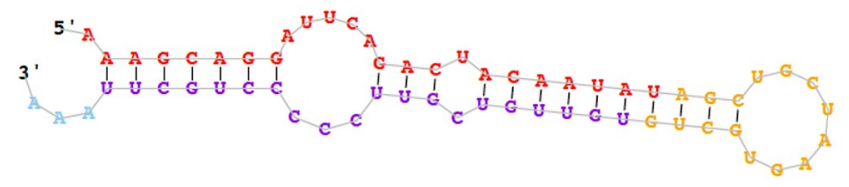

B. hsa-miR-nov7

(i)

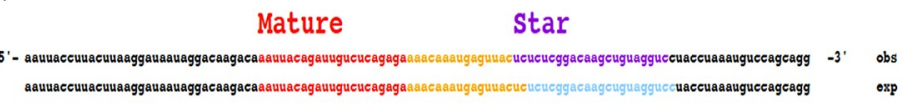

(ii)

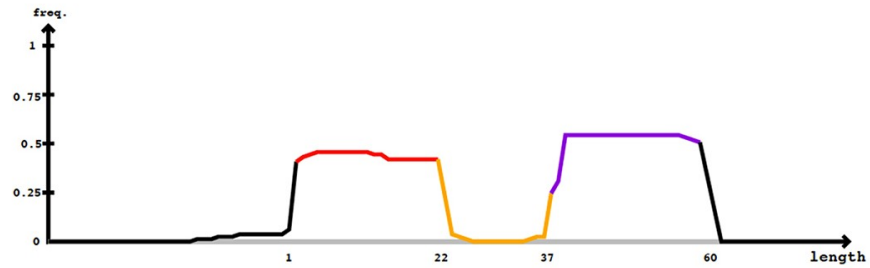

(iii)

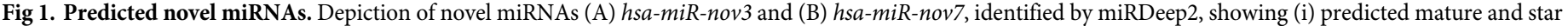
sequences, exp, probabilistic model expected from Drosha/Dicer processing and obs, observed sequences from sequencing data (ii) density plot for read counts for mature and star sequences as well as (iii) miRNA secondary structure.

https://doi.org/10.1371/journal.pone.0253361.g001 
Fig 8 is missing part B. The authors have provided a corrected version here.

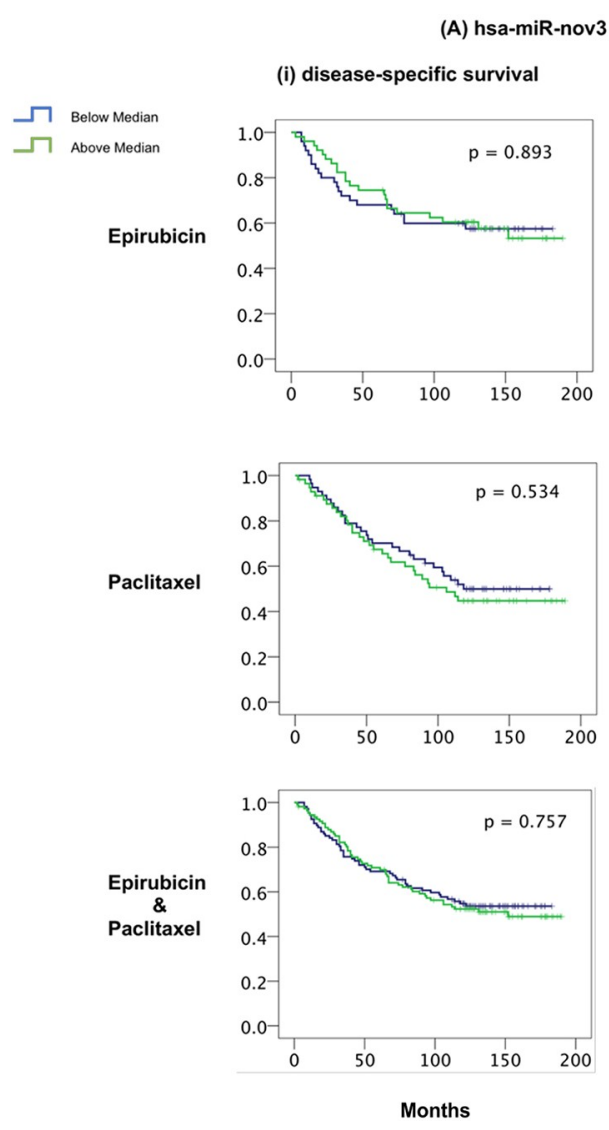

(ii) relapse-free survival
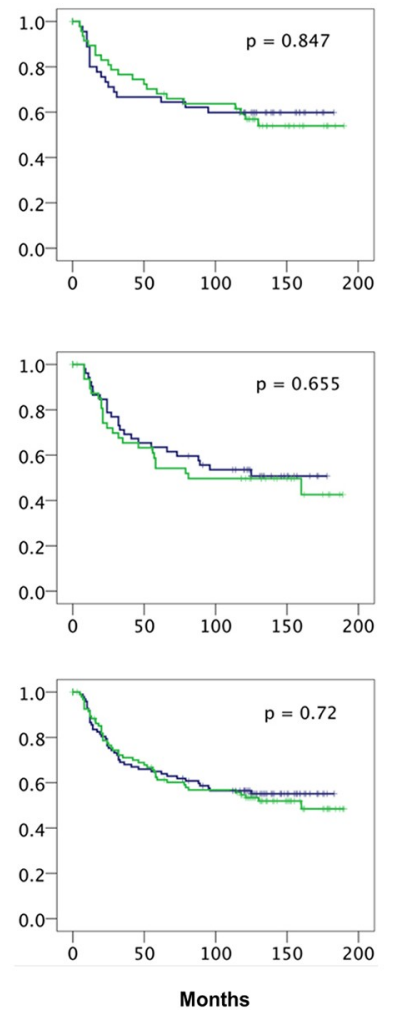

(B) hsa-miR-nov7

(i) disease-specific survival
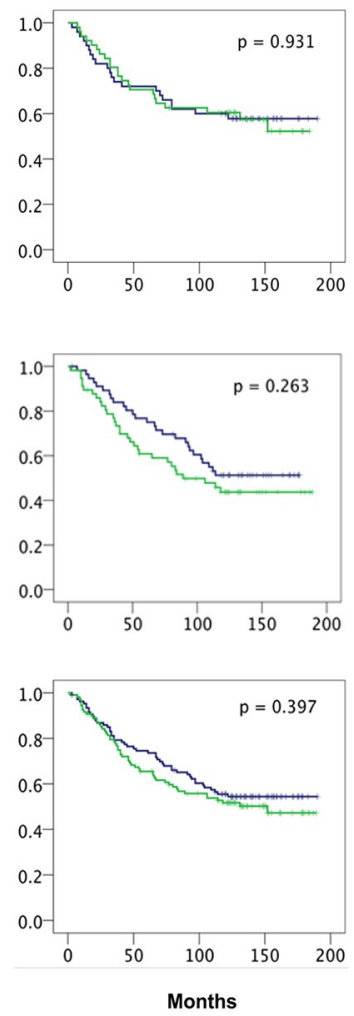

(ii) relapse-free survival
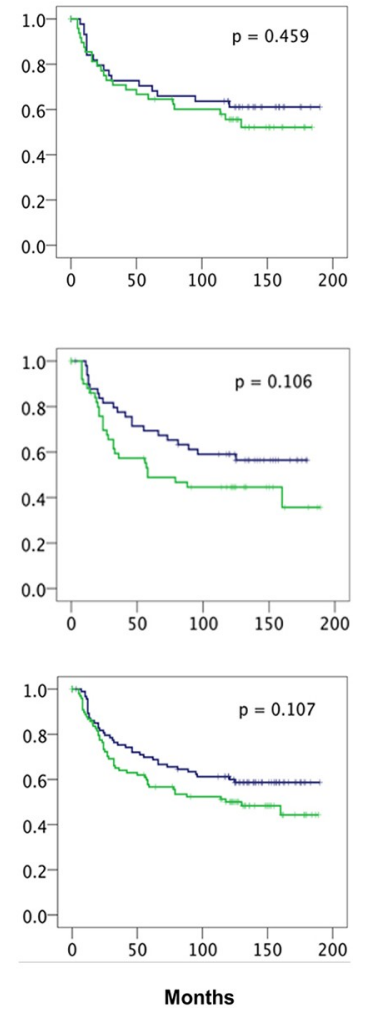

Fig 8. miRNAs and breast cancer survival. Kaplan-Meier curves showing (i) disease-specific and (ii) relapse-free survival of locally advanced breast cancer patients treated with epirubicin or paclitaxel monotherapy in the neoadjuvant setting (study 1), with respect to expression levels of (A) hsa-miR-nov3 and (B) hsa-miR-nov7 on all samples.

https://doi.org/10.1371/journal.pone.0253361.g002

\section{Reference}

1. Poduval D, Sichmanova Z, Straume AH, Lønning PE, Knappskog S (2020) The novel microRNAs hsamiR-nov7 and hsa-miR-nov3 are over-expressed in locally advanced breast cancer. PLoS ONE 15(4): e0225357. https://doi.org/10.1371/journal.pone.0225357 PMID: 32298266 